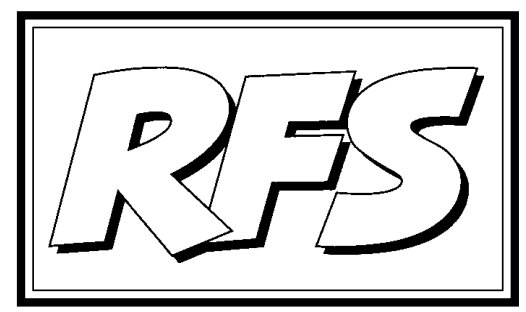

Revista de Fomento Social, 57 (2002), 629-652

\title{
Conciliación de la vida privada y la actividad profesional: La alternativa del teletrabajo ${ }^{1}$
}

Procure V. Sría. en el futuro atender de tal modo a las obras de caridad fraterna, que no se tome demasiado afán ni ansiedad, sino que trabaje con moderación, contentándose de haber hecho de su parte lo que convenía y tome el resultado, sea cual fuere, suavemente, como quien espera que Dios nuestro Señor suplirá en lo que nosotros faltamos.

SAn Ignacio de Loyola

* Profesor del área de Recursos Humanos del departamento de Economía General, Ciencias jurídicas y Sociología de la Facultad de CC.EE. y Empresariales-ETEA adscrita a la Universidad de Córdoba.

1 José Antonio Ariza es también autor de un libro que versa sobre este asunto titulado El reto del equilibrio: vida personal y profesional. Una guía práctica para decidir cómo emplear su tiempo. Editorial Desclée de Brouwer, Colección ETEA, Bilbao, 2002. 


\section{Vida personal y profesional: Un equilibrio necesario}

La sociedad de siglo XXI exige a sus ciudadanos esfuerzos de adaptación cada vez más intensos debido a la profundidad, intensidad y rapidez de los cambios que se están produciendo en una etapa de aceleración histórica desconocida hasta el momento. Este suceso está moldeando una sociedad cada vez más competitiva ${ }^{2}$, donde el trabajo se convierte en el centro neurálgico del ser humano, hasta tal punto de valorar a éste, no por lo que es personalmente, sino por lo que es profesionalmente. El trabajo confiere prestigio social y da plenitud y significado a la vida. Según Tomás Melendo (1994) ostenta una nobilísima valencia antropológica: se configura como uno de los principales factores del perfeccionamiento estrictamente personal, como un utensilio privilegiado para el acrecentamiento y la maduración interiores de la persona humana, considerada precisamente como persona (p. 48).

Bajo este prisma, existe una predisposición a creer queel éxito profesional es más importante que la vida personal. Así parece desprenderse de una reciente encuesta del CIS (2000) según la cual, el 64,0\% de los españoles opinan que el trabajo es la actividad más importante de una persona. En este marco de referencia no es de extrañar que el conflicto entre la vida personal y la dedicación a la carrera profesional sea un problema cada vez más extendido en los países desarrollados. La importancia de este asunto ha llegado a la Cumbre Europea de Estocolmo de marzo de 2001, donde se establecieron una serie de criterios esenciales para mejorar la calidad del trabajo y el empleo. Con este marco de referencia, Paoli y Merllié (2001) establecieron los pilares fundamentales que sustentan la calidad del trabajo y el empleo en la Unión Europea (ver figura 1). Como se puede apreciar, la conciliación de la vida laboral y familiar se configura en uno de los asuntos prioritarios de las políticas de empleo de la Unión Europea, un vector crítico sin el cual las restantes medidas no obtendrían los resultados previstos.

2 Algunos autores, como Friedman y Rosenman (1974), ya advirtieron que la conducta crónica e incesante por lograr cada vez más cosas en menos tiempo es un comportamiento socialmente aceptado e incluso elogiado. 


\section{Figura 1. Calidad del trabajo y empleo en la Unión Europea}

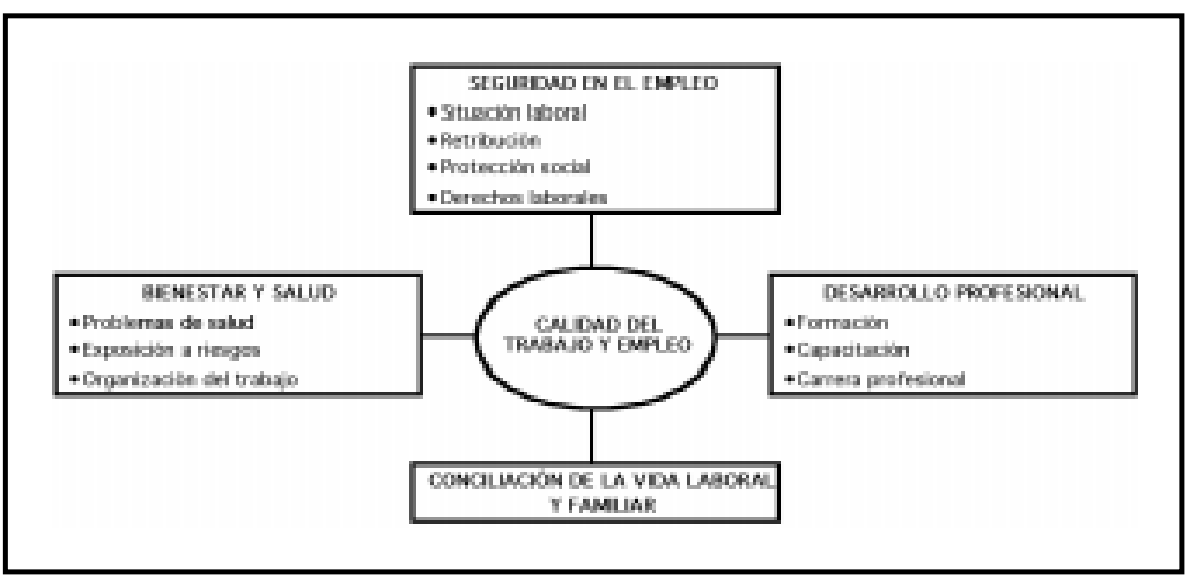

Fuente: elaboración propia a partir de Paoli y Merllié (2001).

A pesar de la difusión de mensajes socialmente aceptables, así como del surgimiento de iniciativas provenientes del sector público y privado para apoyar la conciliación, la realidad dictada por el mercado laboral dibuja un panorama poco esperanzador ${ }^{3}$. El cuadro previsto sugiere que en los próximos años la balanza vida personal/profesional se incline hacia esta última. En este sentido existen señales de alerta más que evidentes: aumento del número de horas de trabajo real, trabajo nocturno, en fin de semana, con jornada partida, horarios irregulares, etc. Las consecuencias aparecen de modo dramático en las estadísticas: fatiga, jaquecas, dolores de espalda o dificultad para conciliar el sueño, entre otras. Todo ello concurre en un aumento del número de casos de adicción al trabajo ${ }^{4}$ y en la propagación de las llamadas enfermedades emergentes como la depre-

3 Para profundizar en este asunto puede consultarse nuestro anterior trabajo publicado en el número 225 de esta revista y titulado El dilema del equilibrio vida personal versus vida profesional: un enfoque de recursos humanos.

4 Estos casos son cada vez más frecuentes. Para los adictos al trabajo, la actividad profesional constituye el eje sobre el que gira la vida y le confiere todo su significado. Utilizando las palabras de Melendo (1994), el trabajo se enfoca casi como una droga, como instrumento de satisfacción y de afirmación de sí mismo, y como medio de eludir otras obligaciones todavía más apremiantes, como pudieran ser las familiares o las de amistad (p. 153). 
sión, el estrés o el desgaste profesional ${ }^{5}$.

En este contexto, a priori se podría suponer que el teletrabajo es una poderosa herramienta para compaginar la actividad profesional con la vida personal y familiar. Un informe de la Comisión Europea se manifiesta en estos términos: el teletrabajo hace al trabajador más independiente al ser responsable de la planificación de su propio tiempo, del mismo modo que le permite equilibrar su vida personal y profesional (Unión Europea, 2000). El Informe Dahrendorf, elaborado por un grupo de expertos para la OCDE en 1985-86, también se pronuncia en relación con la influencia de la tecnología en el asunto que nos ocupa: la innovación tecnológica en parte permite y en parte exige una redistribución más flexible del tiempo de trabajo e incluso del lugar de trabajo y de las formas de organización de éste. Sus incidencias sobre el ritmo de vida familiar pueden ser a veces negativas, pero a largo plazo pueden contribuir a modificar la relación entre vida de trabajo y vida privada (citado en Camacho, 1987, p. 354).

En los próximos apartados profundizaremos en el teletrabajo, incidiendo en los efectos - positivos o negativos- que éste puede originar en la distribución del tiempo privado y profesional.

\section{Teletrabajo: El cisma del concepto}

El desarrollo tecnológico ha propiciado la aparición de gran cantidad de negocios vinculados a la nueva economía y caracterizados por el uso de Internet como medio para lograr sus objetivos: dar a conocer los productos de la empresa, mejorar la relación con los proveedores, optimizar la comunicación interna, etc. Los cambios que se están produciendo se comparan con los acaecidos en la Revolución Industrial. No obstante, la rapidez y las incertidumbres sobre dónde se encuentran los límites a este desarrollo vertiginoso, hacen pensar que aquella era insignificante frente a esta nueva Revolución Tecnológica.

En un contexto de efusión de los e-business, el trabajo y la forma de desarrollar éste no pueden quedar al margen de tales transformaciones. Aunque las previsiones no siempre son homogéneas, los mensajes más frecuentes apuntan a una menor estabilidad e intensidad de la relación

5 Las estadísticas laborales indican que el estrés es, en la actualidad, la segunda causa de absentismo laboral en España. 
laboral, que se traducirá en aspectos como horarios de trabajo flexibles con tendencia a ser más cortos, contratos o relaciones temporales, variedad de tareas, posibilidad de trabajar desde el propio domicilio o con gran movilidad residencial, etc. Así se manifiesta Laporta (2000): variabilidad, flexibilidad, movilidad, versatilidad, creatividad, competitividad, etc. son loscaracteres que van a definir el trabajo del futuro (p. 24). Es incuestionable que las nuevas tecnologías están modificando el estilo de vida y la forma de organización del trabajo de muchos ciudadanos ${ }^{6}$. Para hacernos una idea del impacto que los negocios vinculados a la red tienen en la economía actual hay que señalar que, según el informe de la Comisión Europea titulado Oportunidades de empleo en la Sociedad de la Información (1998), estas empresas son las principales creadoras de empleo en la Unión Europea.

Pero no es suficiente con introducir la tecnología, la piedra angular que determinará el éxito o fracaso en la nueva economía son las personas y el conocimiento asociado a ellas. Los beneficios tecnológi cos sólo se multiplicarán si su introducción está acompañada de nuevas formas de organización del trabajo. Haciendo referencia de nuevo al informe de la Comisión Europea podemos afirmar que en la economía global, que funciona conectada en redes, se están rompiendo las barreras de tiempo, distancia y localización. Las empresas han de ser cada vez más ágiles para aprovechar las oportunidades de la vertiginosa era de la información. Esto requiere modos de trabajar nuevos, más flexibles y adaptables, con menos jerarquía, con más trabajo en equipo, con trabajadores cualificados en varios ámbitos y con más autonomía individual ${ }^{7}$.

El teletrabajo ocupa un papel destacado en este contexto donde afloran nuevas formas de organización del trabajo al amparo de las tecnologías de la

6 Según Casado (2001), en la sociedad de la información y/o economía del conocimiento, la tecnología es consustancial con el hecho económico y social, hasta el punto que generalmente lo conforma y determina. Este autor considera que el teletrabajo es una de las formas más genuinas y "normales" de vivir y producir en la nueva sociedad y/o nueva economía global, interconectada, virtual y digitalizada.

7 Algunos estudios realizados en el ámbito europeo sugieren que el ritmo de introducción de las nuevas tecnologías provocará que, en los próximos años, la mayoría de las herramientas tecnológicas serán diferentes a las que se conocen actualmente. Esta circunstancia ya es apuntada por la Dirección General V de la Unión Europea, que advierte que el $80 \%$ de la población trabajadora ha sido formada hace más de diez años, mientras que el $80 \%$ de la tecnología que se utiliza tiene menos de una década. Esta circunstancia exige un esfuerzo de adaptación permanente. 
red. Ante todo hay que advertir que el grado de implantación del teletrabajo es difícil de delimitar debido, justamente, a la imposibilidad de precisar qué tipos de trabajadores habría que incluir en el recuento. Además, las estadísticas laborales existentes no aportan información relevante en este sentido. Por tanto, las cifras a las que aludimos a continuación hay que asumirlas como referencias que deben ser interpretadas con precaución. Bajo esta premisa, la investigación realizada por Empírica (2000) apunta que el boom del teletrabajo que se manifiesta en otros países de la Unión Europea no ha Ilegado aún a España. Según este estudio, en 1999 había en nuestro país 357.000 teletrabajadores. Esto supone que aproximadamente el 2,8\% de la fuerza de trabajo en España teletrabaja, frente al $6 \%$ de media en Europa ${ }^{8}$. Alemania, con más de dos millones de teletrabajadores, ocupa el primer lugar del ranking en valores absolutos, mientras que en términos relativos se sitúan Finlandia (16,77\%), Suecia (15,17\%), Países Bajos (14,53\%) y Dinamarca $(10,48 \%)^{9}$.

Ante todo, es necesario concretar qué se entiende por teletrabajo. En una primera aproximación debemos advertir que no existe un concepto claro, preciso y riguroso que se haya impuesto de forma generalizada entre los miembros de la comunidad científica. Por el contrario, la ampliación o restricción del vocablo nos lleva a expandir o limitar enormemente las personas que podrían incluirse en la etiqueta de teletrabajadores. En este sentido, resultan esclarecedoras las palabras de Montero (1998) cuando afirma que existen distintas acepciones del término, pudiendo considerar teletrabajo, en senti do estricto sólo a aquellos que trabajan siempre en su hogar, o uniéndolos a aquellos que lo hacen a través de un telecentro, o considerar a todos los que utilizan habitualmente la red informática y otros instrumentos de telecomunicación (p. 206).

De esta manera, Halpern utiliza el término cibertrabajo de una manera reduccionista para referirse a una nueva tipología de trabajo a partir del

8 Este porcentaje contrasta con los datos aportados en J ohnston y Nolan (2002) que cifran la ratio de teletrabajadores en España en el 0,6\%. Esta diferencia puede estar motivada porque estos autores cuantifican únicamente a los teletrabajadores a domicilio que, como veremos a continuación, constituyen tan sólo una parte del conjunto total.

9 Según la III Investigación Europea de Condiciones de Trabajo 2000, realizada entre las quince naciones de la Unión Europea, el Reino Unido es el país con mayor tasa de penetración del teletrabajo: el $10 \%$ de la población activa teletrabaja como mínimo una cuarta parte de su tiempo y dos de cada cien lo hacen a tiempo completo. En España estas cifras son del $4 \%$ y del $2 \%$ respectivamente. 
desarrollo de Internet. Consiste en una modalidad de trabajo a distancia caracterizada por el uso de las tecnologías de la red. Una definición similar es aportada por Adan (2002), cuando precisa que el teletrabajo es una forma de trabajar que hace un uso intensivo de las nuevas tecnologías, distanciado físicamente de la sede organizacional, en un lugar que puede ser el hogar, un hotel, un centro de servicios, etc. El concepto aportado por la Asociación de Empresarios de Campollano (ADECA) insiste en la distancia espacial y en el uso de nuevas tecnologías, al mismo tiempo que se refiere expresamente a la flexibilidad que aporta esta nueva forma de organización del trabajo ${ }^{10}$. La Asociación Francesa de Teletrabajo (AFTT) restringe algo más el concepto, asumiendo que éste puede combinar presencia y ausencia del centro habitual de trabajo, e introduciendo un límite temporal máximo de permanencia ${ }^{11}$. Por su parte, la Asociación Española de Teletrabajo (AET), apuesta por emplear el término e-trabajo ${ }^{12}$, y advierte que pueden existir distintos tipos

10 Según ADECA, el teletrabajo es una forma de trabajo que no requiere la presencia física del trabajador en la propia empresa. Es decir, el trabajador puede realizar sus tareas de manera flexible en el lugar que prefiera. Para ello, es fundamental que la empresa tenga bien desarrollados los sistemas informáticos y tecnológicos, para que el teletrabajador esté en permanente conocimiento de lo que ocurre en su empresa y pueda a su vez, comunicarse con ella. Gray, Hodson y Gordon (1995) también insisten en la flexibilidad como nota característica del concepto. Estos autores consideran que el teletrabajo es una forma flexible de organización del trabajo, que consiste en el desempeño de la actividad profesional sin la presencia física del trabajador en la empresa durante una parte importante de su horario laboral. Engloba una amplia gama de actividades y puede realizarse a tiempo completo o parcial. El desarrollo de la actividad implica el uso frecuente de métodos de procesamiento electrónico de información, así como algún medio de telecomunicación para el contacto entre el teletrabajador y la empresa.

11 La AFTT califica el teletrabajo como aquel que se realiza fuera del lugar habitual, utilizando alguna de las tecnologías de telecomunicación, y por lo menos uno o dos días a la semana. Weijers, Meijer y Spoelman (1992) también establecen un condicionante temporal, al considerar que el teletrabajo es aquel trabajo que, como resultado de la aplicación de la tecnología de la información y de la comunicación, está separado de la localización del empleado por lo menos el $20 \%$ de las horas de trabajo.

12 La AET (2002), en su estudio Situación y problemática global del teletrabajo. El e-trabajo: propuestas de solución, propone el uso de este término, ya que el concepto de e-trabajo no es simplemente "trabajar a distancia", sino algo más profundo que incluye el hecho de "trasladar las mentes, no los cuerpos", creándose un espacio tácito de trabajo (una oficina virtual) donde pueden coexistir todos los componentes, virtualmente reunidos (miembros de un equipo, clientes, proveedores, etc.) que permite la aparición de nuevas relaciones (trabajo en red), posibilidad que facilita la configuración de organizaciones distribuidas. Las TICs permiten un nuevo modo de trabajar y relacionarse muy rico y complejo, donde la palabra teletrabajo se manifiesta insuficiente para reflejarlo. 
de vinculación -laboral o no- de los teletrabajadores con la empresa. Así, la AET considera al teletrabajo como aquella actividad realizada fuera del espacio físico de la empresa que desempeña un trabajador, ejerciendo sus conocimientos al servicio de la misma en régimen de contrato en plantilla, autónomo o freelance, arrendamiento de servicios, etc.

Como puede apreciarse en la Figura 2, las aproximaciones al concepto inciden en distintos aspectos como la distancia con respecto al centro de trabajo habitual, el uso de las tecnologías de la red, etc. En nuestra opinión, es posible que la definición más precisa del término, amplia necesariamente, sea la aportada en el informe eWork 2000, elaborado por Johnston y Nolan (2000) y patrocinado por la Comisión Europea. Según estos autores, el e-trabajo consiste en la realización del trabajo en un lugar distinto del centro habitual (aunque no excluye que el teletrabajad or haga presencia de manera esporádica en la empresa) con el uso de medios telemáticos como instrumento fundamental de trabajo y de comunicación con la empresa. Esta definición incide en los requisitos de distancia, flexibilidad y utilización de herramientas telemáticas, aunque no precisa que éstas sean los recursos de la red.J o hnston y Nolan añaden como característica definitoria del teletrabajo la relación contractual, que puede ser tanto por cuenta propia como ajena, a diferencia de otros autores que limitan el término a los teletrabajadores con vinculación laboral con la empresa. Por último, estos autores se alejan de las tesis defendidas por otros expertos con relación a la necesidad de formalizar el teletrabajo en un contrato específico, lo que resulta difícil, sobre todo teniendo en cuenta que muchos teletrabajad ores no son conscientes de tal condición ${ }^{13}$.

13 Las respuestas a una pregunta del Centro de Investigaciones Sociológicas (CIS, 1998) sobre ¿qué actividad (de una relación propuesta) se aproxima más a su opinión sobre el «teletrabajo»? indican el desconocimiento que existe sobre el tema: un $29 \%$ respondió que consistía en trabajar desde casa; un 17\% pensaba que suponía trabajar a través del televisor; y otros porcentajes más pequeños se asignaron a impresiones como trabajar cuando uno quiera, trabajar menos horas o trabajar sin ser visto. 


\section{Figura 2. Factores a considerar en la definición de teletrabajo}

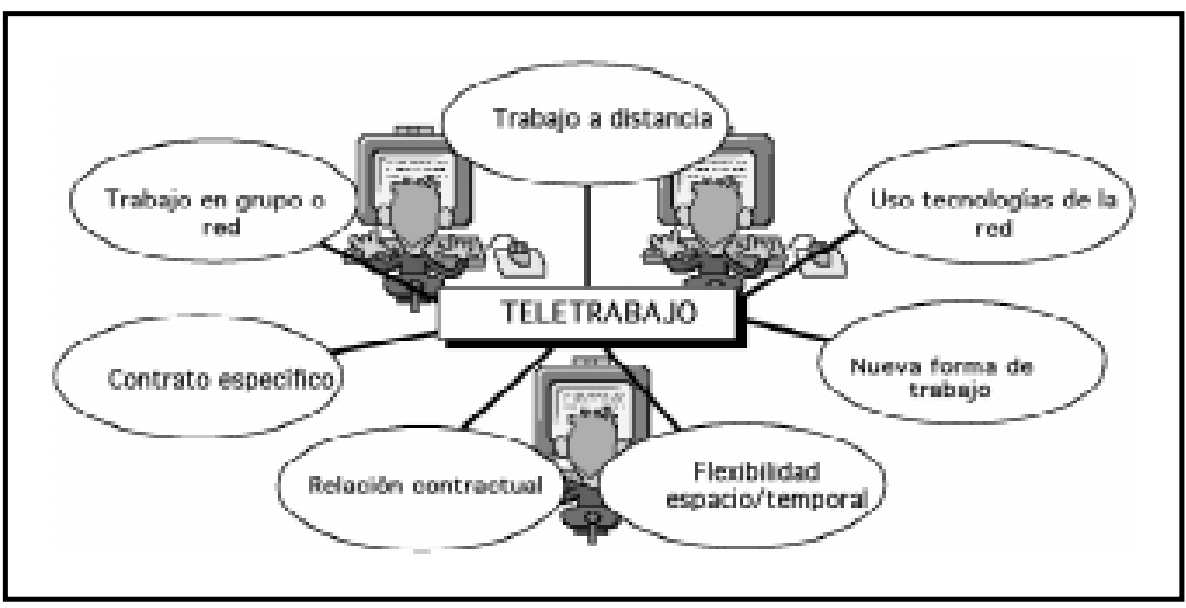

Fuente: elaboración propia.

Como es de suponer, la ausencia de un criterio homogéneo en relación con el tema que estamos tratando, condiciona enormemente cualquier análisis posterior. Pese a esta dificultad, a continuación nos referiremos a los distintos tipos de teletrabajadores que pueden existir.

\section{Tipología de teletrabajadores}

Los principales tipos de teletrabajadores que existen en el mercado laboral están determinados por la definición aceptada. De esta manera, siguiendo las recomendaciones del estudio EcaTT, se podría afirman la existencia de las siguientes modalidades de teletrabajo ${ }^{14}$ :

En primer lugar, el teletra bajo a domicilio sería aquel realizado desde el domicilio particular al menos un día completo por semana. Además, se caracteriza por el uso del ordenador en la realización del trabajo y por la comunicación por teléfono, fax, e-mail, etc. con los superiores y compañeros. Esta modalidad de teletrabajo puede desarrollarse por cuenta propia o 14 Este informe, elaborado por Empirica (2000), trata sobre el futuro del comercio electrónico
y analiza las principales tendencias del teletrabajo en Europa. 
ajena. El estudio EcaTT identifica dos categorías de teletrabajadores a domicilio: por un lado, los perma nentes, aquellos que trabajan más del $90 \%$ de la jornada laboral desde su domicilio y, por otro, los circunstanciales, que trabajan menos del $90 \%$ de su jornada laboral total desde casa, pero más de un día completo por semana ${ }^{15}$.

Los centros de teletrabajo o telecentros son establecimientos que facilitan un lugar de trabajo a empleados de una o varias empresas, u ofrecen servicios de telecomunicación a clientes remotos. El trabajador se desplaza a estos telecentros en lugar de acudir a las instalaciones de la empresa. Según el estudio EcaTT, existen dos tipos de telecentros: oficinas sa télites (instalaciones de la organización que, estando distantes del centro principal de trabajo, se ponen a disposición de los teletrabajadores) y telecentros de proximidad (similares a las oficinas satélites excepto por el hecho de que las dependencias y equipos se ponen a disposición de cualquier empresa que quiera alquilar los servicios que se ofrecen).

Por último, el teletrabajo móvil, donde se incluyen aquellos teletrabajadores que trabajan al menos diez horas a la semana fuera de su domicilio y de su centro habitual, por ejemplo, en las dependencias de sus clientes. Además, deben utilizar una conexión informática para desempeñar su trabajo y comunicarse con la empresa ${ }^{16}$.

Como se puede comprobar en esta clasificación se utilizan dos criterios fundamentales para considerar a una persona como teletrabajador: Ia distancia con relación al centro de trabajo habitual y la duración temporal de ese alejamiento. No obstante, este último elemento sólo es vinculado al trabajo que se ejecuta desde el domicilio particular, cuando, desde nuestro punto de vista, sería aplicable también al teletrabajo móvil y a los centros de teletrabajo. Por consiguiente, se podrían categorizar los tipos de teletrabajadores en una matriz de nueve situaciones posibles (ver Figura 3).

15 Los teletrabajadores que, aunque realizan parte de su jornada de trabajo desde el domicilio, no reúnen el requisito de trabajar como mínimo un día completo a la semana se denominan suplementarios u ocasionales.

16 La clasificación realizada por Benhamou y Saal en 1998 no considera esta última categoría de teletrabajo. Estos autores distinguen entre: tra bajo en casa como la forma más popular, que consiste en trabajar desde el propio domicilio al gunas o todas las jornadas laborales; oficina sa télite, especie de sucursal independiente de la sede central a la que acuden los empleados; y centro laboral vecinal o telecentro donde se comparten unos recursos propios o ajenos en la propia localidad de residencia del teletrabajador. 
En cualquier caso el teletrabajo implica mayor flexibilidad, facilitando de esta manera la conciliación, pero al mismo tiempo es incuestionable que las posibilidades de armonizar la vida personal con la profesional aumentan a medida que éste se aproxima a la categoría de permanente y se permite su desempeño desde el domicilio particular.

\section{Figura 3. Tipos de teletrabajadores}

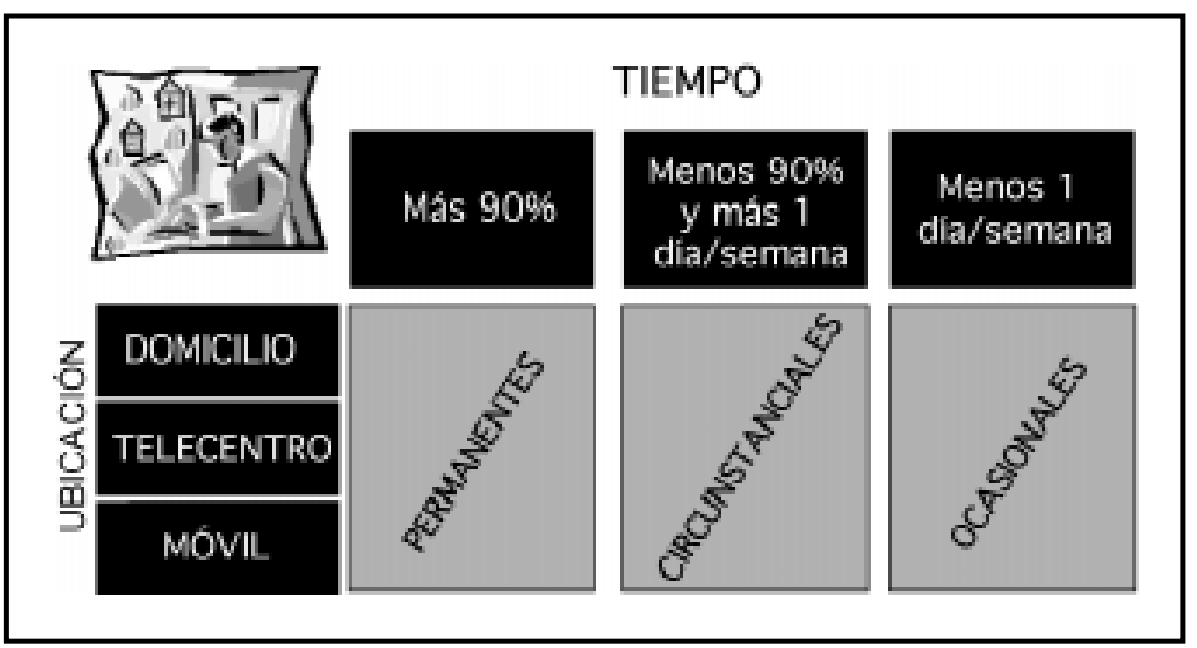

Fuente: elaboración propia.

\section{Principales consecuencias de la implantación del teletrabajo: Afectados versus beneficiados}

Las cualidades que acompañan al teletrabajo son objeto de controversia y polémica entre los estudiosos del tema. Es posible que la investigación empírica en estesentido aún no esté suficientemente desarrollada como para obtener evidencias aceptadas por la comunidad científica. La clasificación que aportamos a continuación se agrupa en cuatro categorías ${ }^{17}$ en función,

17 Las conclusiones obtenidas están fundamentadas en la revisión de los trabajos realizados por los siguientes autores: ADECA, Prieto, Simón, y Vriens, Huws (1984), Prieto, (1989a), Prieto, (1989b), Kaye, (1992), Carrasco y Salinas (1994), Videgain (1995), Tobio y Casado (1995), Fernádez y Miguel (1996), Traverso, Roman, y Alfaro (1999), Steward (2000). 
por un lado, de que supongan un beneficio o una pérdida y, por otro, de los afectados: trabajador o empresa ${ }^{18}$.

\section{A. Efectos positivos para el trabajador y la empresa}

De modo habitual, al teletrabajo se le han atribuido connotaciones negativas (Heck y otros, 1995), sin embargo, a finales del siglo XX, esta especulación ha cambiado gradualmente (Dunn, 1999; Ulfelder, 1999; Morgan, 1999).

Las excelencias del teletra bajo para el empleado se pueden agrupar en torno a tres categorías: aumento de la renta personal, mayor significación del trabajo y mejora en la calidad de vida individual.

- Aumento de la renta disponible. Desde un punto de vista estrictamente económico, el teletrabajo genera un incremento de ingresos y una disminución de gastos. El aumento de los ingresos tiene su origen en el ensanchamiento del mercado laboral. La supresión de las barreras geográficas permite al teletrabajador acceder a un mercado sin fronteras y, en algunos casos, prestar sus servicios a varias empresas. La reducción de los gastos personales se atribuye al ahorro en desplazamientos, desayunos, comidas fuera del domicilio y a una menor necesidad de inversión en vestuario. El trabajador también puede salir beneficiado por disponer de herramientas informáticas para su uso personal. Además, si trabaja por cuenta propia podrá desgravar fiscalmente ciertos gastos que se originan en el domicilio particular: luz, teléfono, alquiler, etc.

- Mayor significado de la actividad laboral. Atendiendo a la organización del trabajo, el teletrabajador dispone de mayor flexibilidad, auto-

18 Algunos autores consideran un tercer actor en esteescenario: la sociedad en su conjunto. Los principales efectos positivos del teletrabajo en ésta serían los siguientes: a) menor necesidad de desplazamiento que, por un lado, reduciría los problemas de circulación y los accidentes de tráfico, y, por otro, disminuiría el consumo energético y la contaminación; b) mayor flexibilidad que se traduce en nuevas oportunidades de empleo para ciertos colectivos como discapacitados, mujeres, persona mayores, etc., al mismo tiempo que facilita el desarrollo de zonas rurales y el abaratamiento del precio de la vivienda en los núcleos urbanos. Entre otros, los principales inconvenientes del teletrabajo para la sociedad son los siguientes: necesidad de una nueva regulación laboral y fiscal, ciertos sectores de actividad podrían verse afectados negativamente (inmobiliarios, restauración...), posibilidad de nuevos desequilibrios territoriales dependiendo de la dotación de infraestructuras tecnológicas, desplazamiento del trabajo a países con menores costes salariales, deshumanización social, etc. 
nomía e independencia para organizar su actividad. Si a ello se añade que, en general, el teletrabajo exige un mayor número de conocimientos, aptitudes y habilidades, el resultado será un trabajo más enriquecedor y gratificante.

- Mejor calidad de vida. A esta conclusión se llega por diferentes motivos. Por un lado, se dispone de más tiempo libre como consecuencia de un ahorro en desplazamientos, interrupciones, contactos sociales en el lugar de trabajo, etc. ${ }^{19}$ También se puede planificar el horario con mayor flexibilidad, ajustándolo al ritmo biológico personal (por ejemplo, muchas personas aumentarían su rendimiento notablemente si pudieran trabajar por la noche). En este contexto resulta más fácil armonizar la vida personal con la profesional, lo que genera un aumento de la motivación y la satisfacción en general, y un descenso de los niveles de estrés. Por otra parte, el trabajador fijará su residencia sin estar condicionado por la localización física de la empresa, y puede acondicionar su entorno laboral en función de sus necesidades, gustos y apetencias particulares. Por último, el trabajar desde casa evita tener que cumplir con ciertos formalismos que forman parte del acerbo cultural de la empresa.

Los beneficios del teletrabajo en las empresas que lo han implantado también son numerosos. Éstos pueden clasificarse en dos grandes apartados. Por un lado, se espera mejorar el resultado económico, mientras que, por otro, se dispone de una estructura más orgánica y de una política de personal más flexible.

- Mejora de los resultados empresariales. El aumento de los márgenes empresariales es consecuencia de unos mayores ingresos, acompañados de una reducción de costes. Los primeros crecen porque la cantidad y calidad del trabajo es mayor. El rendimiento aumenta forzosamente cuando se producen ahorros de tiempo en los desplazamientos, se reduce el absentismo, existen menos interrupciones y mayor concentración, motivación y satisfacción laboral ${ }^{20}$. La contracción de

19 Gray, Hodson y Gordon (1995) estiman que un oficinista medio pasa 700 horas realizando actividades improductivas, en las que se incluyen los desplazamientos al trabajo. Estos autores aseguran que la instauración del teletrabajo provocaría una mejora en el tiempo disponible, de tal forma que el tiempo liberado se podría emplear de manera más provechosa con fines recreativos en vez de malgastarlo en autopistas y aeropuertos (p. 219).

20 Algunos autores defienden que los aumentos de productividad del teletrabajo tienen un 
costes se materializa tanto en la parte fija como en la variable por los siguientes motivos: ${ }^{21}:$ a) menor inversión en instalaciones y sus gastos asociados: amortizaciones, mantenimiento, alquileres, etc.; b) reducción de la factura de luz, teléfono, conexión a Internet, etc. (aunque por otra parte, la empresa tendrá que financiar -en su totalidad o en partelos gastos que los trabajadores tengan por estos motivos en su domicilio);c) menor importe de la retribución no salarial que la empresa abona en concepto de gastos de locomoción, vales de comida, adquisición de prendas de trabajo, compensación por gastos de traslado, etc.; y d) si el trabajador presta sus servicios por cuenta propia o trabaja para más de una empresa es posible ahorrar una parte o la totalidad de las cuotas a la Seguridad Social.

- Estructura más orgánica y política de gestión de recursos humanos más flexible. El teletrabajo provoca que la estructura organizativa esté más descentralizada desde un punto de vista físico, así como de gestión. La supervisión clásica, fundamentada en la desconfianza y el control, se transforma en una supervisión virtual, donde el teletrabajador asume mayor responsabilidad individual en relación con los resultados de su trabajo. Por otra parte, las tareas suelen estar definidas deun modo más difuso, lo que enriquece la variedad y calidad de las aportaciones de los empleados. Todo ello genera una organización más plana, con menos niveles jerárquicos, que reduce el coste de la estructura y favorece la coordinación, y donde resulta más fácil crear nuevos puestos de trabajo, ya que la inversión necesaria es menor.

Desde la perspectiva de la política de gestión de recursos humanos, hay que indicar que en estas empresas la organización de las tareas es mucho más flexible. Ante incrementos puntuales de la demanda se puede acudir a la subcontratación sin que existan limitaciones o barreras geográficas. Además, la flexibilidad se plasma en la cantidad y forma de distribución de las horas trabajadas (horas extras, turnos, horario flexible...), en el tipo de

substrato psicológico. Sin embargo, estamos de acuerdo con Montero (1998) cuando afirma que hay personas más o menos trabajadoras, pero sólo por cambiarles las herramientas de trabajo, normalmente, no les cambiará su personalidad. Lo trabajador que una persona sea pertenece al terreno psicológico de la persona y sus motivos e implicaciones se suelen quedar en ese ámbito (p. 214).

21 Algunos autores como Fernández y Miguel (1996) consideran erróneamente quela reducción de costes se concentra únicamente en los de naturaleza fija. 
vinculación laboral -o no- (trabajo temporal, subcontratación, jornada parcial, trabajo a puesto compartido, etc.) y en la flexibilidad en el contenido del trabajo y su retribución. El sistema de compensación debe cambiar de enfoque, vinculando la retribución al rendimiento individual, por tanto, el modelo de evaluación del desempeño debe ajustarse a esta circunstancia. La política de reclutamiento y selección también se ve favorecida. Por un lado, el mercado laboral se ensancha, al no tener que restringir la búsqueda al entorno más próximo (o tener que abonar una compensación económica en concepto de tras(ado). Por otro, el teletrabajo facilita la atracción y retención de profesionales excelentes, aquellos que aportan talento y aumentan el capital intelectual de la organización. Estas personas, conscientes de su valía profesional y con niveles salariales elevados, demandan fórmulas de compensación individualizadas e innovadoras, donde la retribución económica no constituye el único elemento a valorar. Por consiguiente, la flexibilidad que aporta esta forma de organización del trabajo ayuda a la captación y conservación en la empresa de estas personas. Por ultimo, reseñar que la conflictividad laboral disminuye y las luchas entre agentes internos se diluyen, evitando de esta manera desviar las energías hacia objetivos no coincidentes con los estrictamente organizativos.

\section{B. Efectos negativos para el trabajador y la empresa}

Como acabamos de comprobar, el teletrabajo puede aportar ventajas significativas en el ámbito personal y empresarial. No obstante, esta forma de organización del trabajo también conlleva una serie de problemas que han de evaluarse a la hora de decidir, tanto su implantación, como el modo en que va a ejecutarse. Los principales impedimentos para el trabajador se traducen en mayor precariedad laboral, dificultad para el desempeño del trabajo y un posible empobrecimiento personal.

- Precariedad laboral. En algunos casos, el teletrabajo puede suponer una merma en las conquistas sociales, encontrándose el empleado indefenso anteriesgos no cubiertos o falto dela protección que acompaña al trabajo presencial ${ }^{22}$. Al mismo tiempo, el teletrabajador puede

22 Casado (2001) considera que el teletrabajo no sólo está sirviendo como excusa, en algunas ocasiones, para la contratación precaria de trabajadores, sino también como una potente herramienta de "deslaboralización" del mercado de trabajo. Igualmente añade que cada vez es más frecuente encontrar experiencias de teletrabajo y relaciones laborales encubiertas a través de la figura del falso autónomo. 
encontrar más dificultades para el desarrollo de su carrera profesional, ya que los ascensos y promociones se suelen conceder a aquellas personas que tienen un desempeño visiblemente excelente, y la ausencia habitual del centro de trabajo oscurece la visibilidad. Algunos autores también defienden la tesis de que los teletrabajadores tienen más dificultad para acceder a la formación aunque, en nuestra opinión, esta circunstancia no es determinante. Por último, el trabajador tendrá que afrontar una serie de gastos para poder desempeñar su trabajo desde el domicilio particular. De todos modos, este problema no debería manifestarse, ya que la empresa está moralmente obligada a financiarlos.

- Dificultad para el desempeño del trabajo. Para ser eficaz, el teletrabajador debe reunir, en comparación con un empleado presencial, un conjunto de competencias, aptitudes y cualidades más amplio. Quizás, una de las habilidades más necesarias sea la capacidad de autodisciplina para organizar y planificar el trabajo, ya que no se dispone de una supervisión externa que marque las pautas de comportamiento y controle el progreso y el nivel de logro en los objetivos. El rigor también es fundamental para diferenciar tiempo de trabajo y tiempo personal, sin el cual se podría acabar en un aumento de las horas de dedicación a la actividad laboral, provocando efectos perniciosos sobre el bienestar físico y psicológico del trabajador. Asimismo, para ser más eficaz, éste debe aislar su entorno de trabajo del ambiente familiar, habilitando un lugar libre de perturbaciones e interrupciones no profesionales. Una última barrera al desempeño individual se produce por los problemas técnicos derivados del uso de las nuevas tecnologías (virus, averías, conexiones....), cuya reparación o sustitución se demora cuando el empleado trabaja alejado de las instalaciones de la empresa.

- Empobrecimiento personal. Aunque algunos autores defienden que el teletrabajo no implica un aislamiento del empleado ${ }^{23}$, lo cierto es que el teletrabajador se desvincula en parte de la empresa, produciéndose una desconexión de su realidad social. La autonomía genera ventajas

23 En esta línea se expresa Adan (2002) cuando asevera que las relaciones sociales, lejos de sufrir un deterioro sobre la base de la ausencia de contactos cara a cara, y de la impersonalidad que caracteriza la comunicación electrónica, experimentan en general una mejora tanto en la calidad de los vínculos sociales, como en el número de contactos y la extensión de los mismos. 
desde un punto de vista personal, pero también dificulta el apoyo y la colaboración de los compañeros en momentos puntuales (situaciones de crisis, conflictos laborales, etc.). La ausencia de vida social en la empresa conlleva un empobrecimiento del sujeto como ser social, el trabajo proporciona algunas de las más importantes relaciones sociales suprafamiliares y la inserción del individuo en las exigencias de los intereses colectivos y el bienestar general. Con to do ello, naturalmente, va definiéndose en cada uno la propia identidad social (Laporta, 2000, p. 31). El equilibrio es la clave de una vida plena de significado, de tal modo que muchas personas necesitan complementar su vida privada con unas relaciones sociales derivadas del contacto -permanente u ocasionalcon los compañeros de trabajo.

Los inconvenientes para la empresa -que relativizan las ventajas atribuidas al teletrabajo- pueden agruparse en tres categorías: mayor inversión tecnológica, necesidad de un cambio cultural y una gestión más compleja de los recursos humanos.

- Mayor inversión en tecnología. Desde un punto de vista económico, el mayor inconveniente del teletrabajo radica en la elevada inversión necesaria para la adquisición de los equipos necesarios y para el mantenimiento de éstos.

- Cambio cultural difícil de asumir. Modificar los valores y principios que rigen el funcionamiento de una organización no es fácil. El teletrabajo implica una transformación radical de las prácticas habituales en una organización presencial. Entre otras, habrá que asumir las siguientes: la cadena de mando se hace más plana, dando paso a un aumento dela descentralización; el modelo de gestión tradicional fundamentado en el control debe sustentarse ahora en la confianza, en los resultados, concediendo más libertad de actuación a los empleados; el trabajo en equipo se debe virtualizar, aprovechando las posibilidades que ofrecen las nuevas tecnologías. En definitiva, se debe aceptar un cambio radical en la escala de valores que durante años han regido el funcionamiento de la empresa.

- Gestión de recursos humanos más compleja. La introducción del teletrabajo debe venir acompañada de cambios profundos en los procesos tradicionales de gestión del personal. De esta manera, el reclutamiento y la selección de trabajadores es más compleja, debido a los requerimientos específicos que deben reunir estos: conocimien- 
tos tecnológicos, hábitos coherentes con el teletrabajo, habilidad para autogestionar su tiempo, autocontrol y disciplina, capacidad para trabajar en base a resultados, etc. ${ }^{24}$ Por otra parte, la empresa tendrá que realizar un mayor esfuerzo en formación, si quiere aprovechar todas las potencialidades que ofrece esta nueva forma de organización del trabajo. Además, la comunicación se vuelve más dificultosa, discontinua, al mismo tiempo que necesita de sistemas que garanticen la seguridad y confidencialidad de la información. Por último, los métodos de evaluación del desempeño, y por ende la compensación, deben adaptarse a las nuevas circunstancias, lo que exige una gestión más intensa y proactiva de estas cuestiones.

\section{Principales obstáculos para avanzar desde las trincheras}

En la batalla por armonizar la vida personal con la profesional el teletrabajo se configura como una arma decisiva para el resultado final. A pesar de su calibre, los partes de guerra advierten de su escasa presencia en los frentes de combate, de tal modo que su implantación en la economía actual presenta un alcance muy limitado ${ }^{25}$. El progreso tecnológico y la difusión de la cultura de la red entre un colectivo de población cada vez más amplio, hace pensar que las condiciones para la propagación del teletrabajo vayan mejorando en los próximos años. A pesar de este planteamiento, como comprobaremos a continuación, aún son muchos los obstáculos que frenan una conquista más rápida e incisiva.

La investigación realizada por Empírica (2000) en diez países de la Unión Europea identifica las principales barreras que frenan el desarrollo del teletrabajo. Como puede apreciarse en la Figura 4, en España, los motivos

24 Para profundizar en las cualidades que debe reunir el teletrabajador se puede consultar el trabajo de Inglés y Puig (1998).

25 Las expectativas de crecimiento y generalización están estrechamente vinculadas a una variable crítica en todo este proceso: el desarrollo e implantación en la sociedad de las nuevas tecnologías. En este sentido, los datos de InfoXXI del Ministerio de Ciencia y Tecnología apuntan que en Estados Unidos accede a Internet el 39\% de la población, y en otros países aún más: Canadá (42\%) o Suecia (40\%). El retraso tecnológico de España es patente: a diciembre de 2000 , únicamente el $15,7 \%$ de los hogares españoles estaban conectados a la red. De todos modos, la trayectoria de los últimos años invita al optimismo. Si en 1999, tan sólo tres millones de españoles estaban familiarizados con la red, en 2001 eran ya más de siete millones, con un crecimiento sobre el año anterior del $94 \%$. 
más aducidos para no implantar éste son: el desconocimiento de la dirección (74,43\%); el miedo a la seguridad de la información (73,38\%); la certeza de que va a reducirse la calidad del trabajo y/o su productividad (69,7\%); el temor a una pérdida de control sobre los empleados (65,58\%); y la creencia de que va a suponer un incremento de costes $(65,02 \%)^{26}$. Si se contrastan estos obstáculos con las razones aducidas por la media de los diez países analizados (ver Figura 4), se aprecia que todas estas barreras al desarrollo del teletrabajo son más pronunciadas en España.

\section{Figura 4. Barreras a la implantación del teletrabajo}

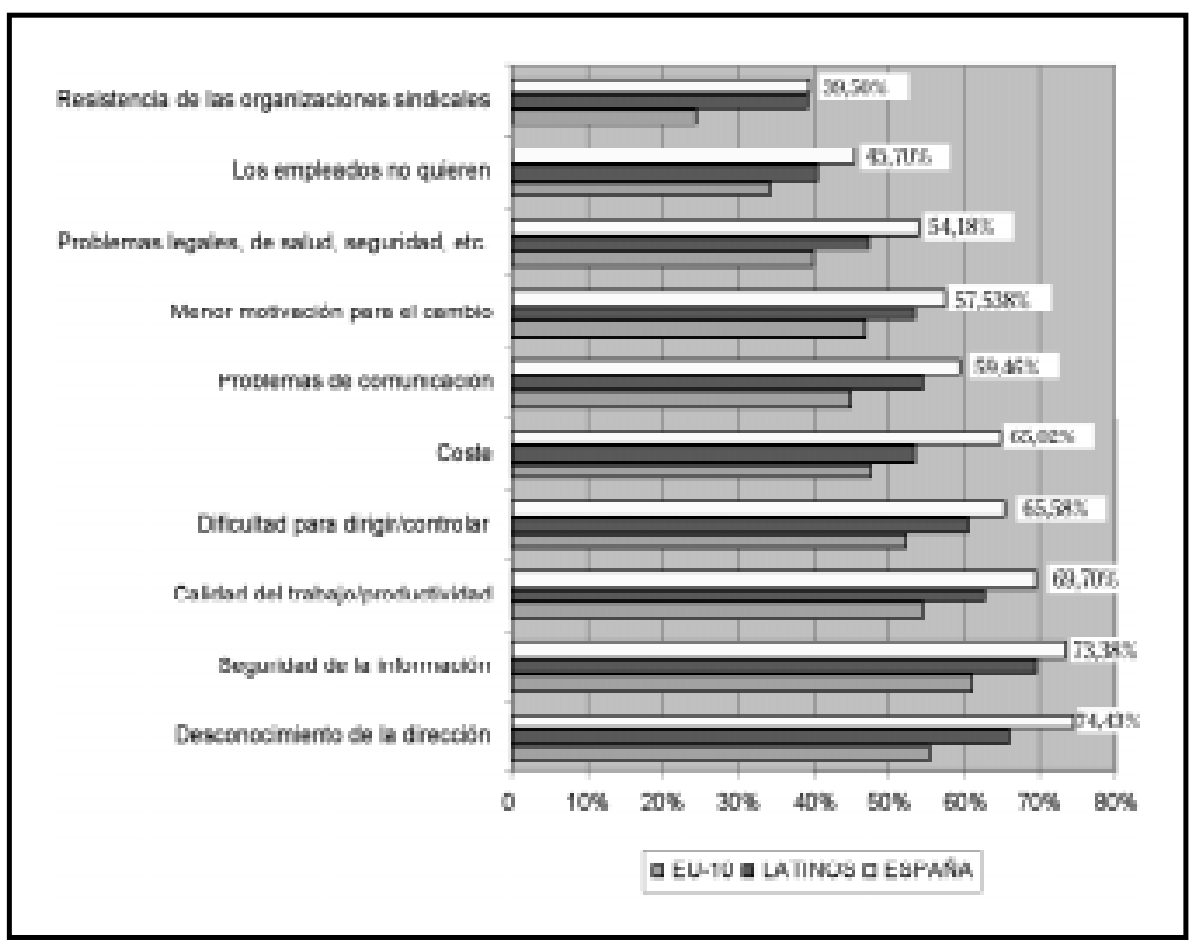

Fuente: elaboración propia a partir de Empírica (2000).

26 El informe Analysis of Home-Based Telework Technology Barriers (2002) elaborado en Estados Unidos identifica las siguientes barreras a la implantación y desarrollo del teletrabajo en este país: 1) la dirección piensa que es difícil mantener los niveles de rendimiento y productividad; 
Dos estudios recientes dan cuenta de la escasa utilización del teletrabajo como herramienta para conciliar la vida familiar con la profesional. Por un lado, la investigación realizada por Poelmans y Chinchilla (2001) con una muestra de responsables de recursos humanos en empresas que actúan en el mercado español, advierte que tan sólo una de cada diez empresas dispone de una política proactiva para mejorar la flexibilidad entre sus empleados. Las medidas adoptadas más comunes son la facilidad para tomar días libres $(90,1 \%)$, las excedencias para el cuidado de los hijos pequeños $(76,3 \%)$ y la posibilidad de trabajar a tiempo parcial $(63,1 \%)$. El teletrabajo sólo es contemplado por un $13,7 \%$ de las empresas analizadas, ocupando una de las últimas posiciones del ranking ${ }^{27}$.

Por otro lado, los resultados del estudio Work \& Life Balance (2001), realizado entre directivos de empresas de 24 países del mundo, atestiguan esta tendencia ${ }^{28}$. Con relación a nuestro país, esta investigación apunta al trabajo a tiempo parcial y a la flexibilidad horaria como principales instrumentos de apoyo a la conciliación. El desconocimiento o el miedo a la pérdida de control sobre el empleado provoca que el teletrabajo sea muy poco utilizado. Las conclusiones del estudio indican que casi la mitad de las empresas consultadas no ofrecen esta opción de flexibilidad laboral a ninguno desus trabajadores. El $45 \%$ la proporcionan a determinados puestos y sólo el $5 \%$ permite a todos sus empleados la posibilidad de teletrabajar.

\section{Conclusión}

Las organizaciones empresariales necesitan empleados lúcidos, activos, descansados, que puedan liberar toda la inspiración y creatividad quellevan dentro. Pese a ello, en muchas ocasiones los profesionales se encuentran

2) elevado coste del equipamiento necesario para el desarrollo del teletrabajo o para la creación de centros de teletrabajo;3) problemas de justicia y equidad entre teletrabajadores y empleados presenciales; 4 ) inquietud acerca de la seguridad de los documentos clasificados; 5) problemas de soledad entre los teletrabajadores; y 6) dificultades de conectividad electrónica con el centro de trabajo.

27 Además, no debemos olvidar que esta investigación fue llevada a cabo entre empresas de tamaño grande, por lo que los resultados pueden estar sesgados por este motivo. El grado de implantación real del teletrabajo en la empresa española debe ser aún más reducido.

28 La investigación obtuvo un total de 2.216 encuestas. En España el estudio estuvo liderado por Creade y se contó con la base de datos de la Asociación de Antiguos Alumnos de ESADE. 
saturados, cansados, exánimes, pulverizados. En estas condiciones, difícilmente podrán dar lo mejor de sí mismos. A esta situación se llega por un contexto laboral donde se enaltece la presencia en vez de la eficacia. De esta manera, la conciliación de la vida familiar con la profesional resulta en extremo complicada.

En este artículo hemos constatado la importancia que a este problema se le concede en las políticas de empleo de la Unión Europea. En relación a esta cuestión, creemos que el teletrabajo puede contribuir de manera positiva a conciliar la vida privada con la actividad profesional. Con esta línea de argumentación, nos hemos aproximado al concepto del teletrabajo y a los principales tipos de teletrabajadores, comprobando la disparidad de criterios y el antagonismo de algunas propuestas, lo que obstaculiza considerablemente el análisis posterior. Precisamente, los efectos del teletrabajo en relación con el tema que nos preocupa -tanto en sentido positivo como negativo- serán más penetrantes a medida que éste se aproxime a la categoría de permanente y se desarrolle desde el domicilio particular.

Por otro lado, también hemos verificado que el teletrabajo, como casi todo en la vida, presenta ventajas e inconvenientes. Ello sugiere la necesidad de hallar un lugar de encuentro que satisfaga las aspiraciones de los empleados, a la vez que se adapta a las posibilidades de la empresa, ya que no todos los trabajos son adaptables por igual a las condiciones y exigencias que impone esta nueva forma de organización de la actividad laboral. Seguramente, el punto óptimo se localiza a medio camino entre el trabajo tradicional, fundamentado en la presencia continua en el centro de trabajo, y el teletrabajo permanente. Parafraseando la «ey del justo medio» enunciada por Kant podemos afirmar que lo saludable está en los medios (un poco de todo) y lo patológico en los extremos (mucho de una sola cosa). Por un lado, los empleados que anhelan trabajar en sus domicilios particulares a tiempo completo, pueden estar ocultando problemas de sociabilidad. Por otro, aquellos que no quieran aprovechar los beneficios que se derivan de trabajar desde el propio hogar, a lo mejor encubren de este modo dificultades personales o una vida demasiado pobre o vacía por completo. Sumergirse en la fosa del mundo personal descompone al ser humano, pero zambullirse en el abismo de la actividad profesional lo pudre también. La inmersión ha de ser controlada, equilibrada, en función de las características personales y los objetivos y expectativas vitales. 


\section{Bibliografía}

AdAn, A.J . (2002). “Repercusiones psicosociales del teletrabajo”. E-trabajo. №2.

ADECA. El teletrabajo. Asociación de Empresarios de Campollano (ADECA). $\varangle$ www.adeca.com>[Consulta: 7 noviembre 2002].

AET. Trabajos y perfil del teletrabajador. Asociación española de teletrabajo (AET). «ttp://www.aet-es.org> [Consulta: 7 noviembre 2002].

Ariza Montes, J.A. (2002). El reto del equilibrio: vida personal y profesional. Una guía práctica para decidir cómo emplear su tiempo. Bilbao. Editorial Desclée De Brouwer, Colección ETEA

Ariza Montes, J .A. (2002). "El dilema del equilibrio vida personal versus vida profesional: un enfoque de recursos humanos". Revista de Fomento Social. № 225, Volumen 57, enero-marzo, pp. 85-100.

Benhamou, E. y SaAL, H.J . (1998). SmartValley Telecommuting Information Guide. <http://www.svi.org/PROJ ECTS/TCOMMUTE/TCGUIDE/telecmmtng_guide.txt> [Consulta: 7 noviembre 2002].

Camacho, I. (1987). "Las disfunciones sociales derivadas de la transformación del trabajo". Revista de Fomento Social. № 168, octubre-diciembre, pp. 339-360.

Carrasco, R. y Salinas, J.M. (1994). Teletrabajo. Madrid. Ministerio de Obras Públicas, Transportes y Medio Ambiente. Secretaría General de Comunicaciones. Dirección General de Telecomunicaciones,

CASADO, R. (2001). ¿Teletrabajo o trabajadores del conocimiento? Gestión del conocimiento.com. $\varangle$ www.gestiondelconocimiento.com/documentos $2 /$ rcasado/trabajo.htm> [Consulta: 7 noviembre 2002].

Centro de Investigaciones Sociológicas, CIS (2000). El año 2000. Boletín no 24, abril-junio. \ttp://www.cis.es / boletin/24/2000.htm> [Consulta: 7 noviembre 2002].

Comisión Europea (2000). Telework Pilot Proyect in the European Commission: External Evaluation Panel Report. Comisión Europea.

CREADE (2001). Work and Life Balance. «www.work-and-life-balance.com> [Consulta: 7 noviembre 2002].

Dunn, K. (1999). "Telecommuting: a tool of the millennium business". Workforce. Vol. 117, no 11 y 22. 
EMPIRICA (2000). Condition for the Development of New Ways of Working and Electronic Commerce in Spain. Empirica, mazo. «www.empirica.com> [Consulta: 7 noviembre 2002].

EmpIRICA (2000). ECaTT, Electronic Commerce and Telework Trends: Benchmarking Progress on New Ways of Working and New Forms of Business across Europe. Empirica. $\varangle \mathbf{w w w . e c a t t . c o m / f r e p o r t / E C a T T - F i n a l - R e p o r t . p d f > ~}$ [Consulta: 7 noviembre 2002].

Fernández, R. y Miguel, J .A. (1996). El teletrabajo: un instrumento de globalización del mercado laboral. Congreso AEDEM. Granada.

Friedman, M. y Rosenman, R. H. (1974). Type A Behavior and Your Heart. Greenwich, Fawcett Publications.

Gray, M., Hodson, N y Gordon, G. (1995). El Teletrabajo. Aspectos generales. Universidad-Empresa, Colección Fórum, p. 63.

HALPERN, J. El Cibertrabajo. Asociación española de teletrabajo (AET). «ttp:/ /www.aet-es.org/docs/cibertrabajo.htm>[Consulta: 7 noviembre 2002].

HeCk, R.; Rowe, B. y Owen, A. (1995). What we know and what we do not know about the "home" and the "work" and the implications of both, en Heck, R., Owen, A. y Rowe, B. (Eds.). Home-Based Employment and Family Life. Auburn House, Westport, pp. 193-228.

Huws, U. (1984). “New Technology Homeworkers”. Employment Gazette. №92, pp. 13-17.

INGLÉs, M. y PUIG, T. (1998). Competencias del teletrabajador. Teletrebages

J ohnston, P. y Nolan, J . (2000). eWork 2000. Status Report on New Ways to Work in the Information Society. Comisión Europea.

J ohnston, P. y Nolan, J. (2002). eWork 2002. Status Report on New Ways to Work in the Information Society. Comisión Europea.

Kaye A.R. (1992) "Collaborative Learning through Computer Conferencing". Berlin The Najaden Papers, vol. 90, Springer-Verlag.

LAPORTA, F.J . (2000). "Incógnitas sobre el trabajo del futuro y el futuro del trabajo". Aedipe, diciembre, pp. 22-31.

Montero, R. (1998). “El teletrabajo: una postura crítica”. Revista ICADE, №44, pp. 205-218. 
Morgan, C. (1999). "Telecommuting accelerates". Computerworld. Vol. 33, № 44.

Paoli y Merllí́ (2001). Tercera Investigación Europea en Condiciones del Trabajo 2000. Fundación Europea para la Mejora de las Condiciones de Vida y Trabajo.

Poelmans, S. y Chinchilla, M. N. (2001). "The Adoption of Family-Friendly HRM Policies. Competing for Scarce Resources in the Labor Market". IESE, Research Paper № 438, junio.

Prieto J.M. (1989a). "Incertidumbre laboral percibida a través de las nuevas formas de empleo y autoempleo". Información Psicológica, 38, 36-41.

Prieto J.M. (1989b). New Ways of Employment and Self-Employment. En B.J . Fallon, H.P. Pfister and J. Brebner, (Eds), "Advances in Industrial and Organizational Psychology," Amsterdam, Elsevier Science Publishers. (p. 285-293).

Prieto, J.M., Simón, C. y VRiens, J. Networking the personnel and the customers inside telecommuterland. Universidad Complutense de Madrid. «ttp:// www.ucm.es/info/Psyap/libros/telework/chapter4.htm> [Consulta: 7 noviembre 2002].

Sosa, S. y Verano, D. (2001). "La influencia del teletrabajo en la dirección y gestión de recursos humanos". Capital Humano. № 144, pp. 70-78.

SteWARD, B. (2000). Changing Times. The meaning, measurement and use of time in teleworking. Londres.Time $\&$ Society. Sage.

TobIo, M. y CASADo, J.M. (1995). “El trabajo del futuro". Capital Humano. № 78, pp. 14-17.

Traverso, J., Roman, M. y Alfaro, A. (1999). "Presencia y consecuencias del teletrabajo en el sector bancario. Un estudio empírico". Dirección y Organización. Enero, pp. 35-43.

Ulfelder, S. (1999). “Extend your reach". Computerworld. Vol. 33, no 44.

VIDEGAIN, J J . (1995). "IBM España, una experiencia europea de teletrabajo". Alta Dirección. № 184, pp. 395-402.

Weijers, T.; Meijer, R. y Spoelman, E. (1992). "Telework remains "made to measure" The large scale introduction on telework in the Nederlands". Futures. Vol. 24, no 10. 